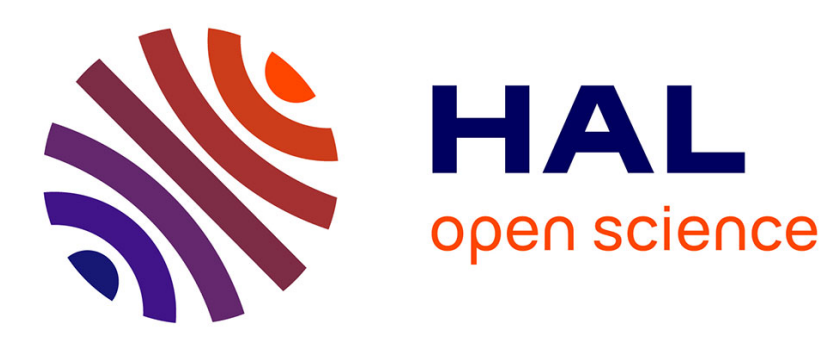

\title{
Reading bits on a CD-ROM without a photodiode
}

Michael Joseph Wishon, A. Locquet, Guillaume Mourozeau, Daeyoung Choi, Sreejith K.R., Aakash Sahai, David Citrin

\section{To cite this version:}

Michael Joseph Wishon, A. Locquet, Guillaume Mourozeau, Daeyoung Choi, Sreejith K.R., et al.. Reading bits on a CD-ROM without a photodiode. IET Optoelectronics, 2017, 11 (5), pp.213-216. 10.1049/iet-opt.2016.0118 . hal-03014515

\section{HAL Id: hal-03014515 https://cnrs.hal.science/hal-03014515}

Submitted on 19 Nov 2020

HAL is a multi-disciplinary open access archive for the deposit and dissemination of scientific research documents, whether they are published or not. The documents may come from teaching and research institutions in France or abroad, or from public or private research centers.
L'archive ouverte pluridisciplinaire HAL, est destinée au dépôt et à la diffusion de documents scientifiques de niveau recherche, publiés ou non, émanant des établissements d'enseignement et de recherche français ou étrangers, des laboratoires publics ou privés. 


\section{Reading bits on a CD-ROM without a photodiode}

ISSN 1751-8768

Received on 12th September 2016 Revised 15th April 2017

Accepted on 5th May 2017

E-First on 21st August 2017

doi: 10.1049/iet-opt.2016.0118

www.ietdl.org

\author{
Michael Joseph Wishon ${ }^{1,2}$, Alexandre Locquet ${ }^{2,1} \bowtie$, Guillaume Mourozeau ${ }^{1,2}$, Daeyoung Choi ${ }^{1,2}$, Sreejith \\ K.R. ${ }^{1,2}$, Aakash Sahai1,2, David Citrin ${ }^{1,2}$ \\ ${ }^{1}$ ECE Department, Georgia Institute of Technology, 777 Atlantic Drive NW, 30332-0250 Atlanta, GA, USA \\ ${ }^{2}$ Unité Mixte Internationale 2958 Georgia Tech-CNRS, Georgia Tech Lorraine, 2 Rue Marconi, 57070 Metz, France \\ 凶E-mail: alocquet@georgiatech-metz.fr
}

\begin{abstract}
The authors demonstrate that the bits of a Compact Disc - Read Only Memory (CD-ROM) can be read without a photodiode (PD). A commercial CD-ROM drive was used and left unmodified except a beam splitter was removed which would route the light to a PD. This allowed for the reflected light to be incident on the laser within the laser package itself. By monitoring fluctuations in the voltage across the laser diode under constant-current injection, it was possible to read and interpret the bits from a spinning CD-ROM.
\end{abstract}

\section{Introduction}

Optical feedback into a laser diode (LD) can induce profound modification to its light-emission properties, and has thus attracted considerable attention over the past four decades starting with applications in velocimeters and position sensors [1-4]. These devices exploit the beating within the cavity at the Doppler frequency of returned light with the light emitted by the LD. The output intensity modulated at the Doppler frequency is measured by a photodiode (PD). However, other properties may also be modified by the feedback. For example, in [5], Lacot et al. exploited feedback-induced changes in the relaxation-oscillation frequency. In [6], a change in self-modulation frequency in a periodic regime was detected via the frequency of the LD terminal voltage $V$. Also, the voltage $V$ can be used to probe the carrier dynamics in the active region of an LD [7-9].

We now focus on direct antecedents of our work. Seko et al. demonstrated 'self-quenching' of an LD in front of which a mirror is placed or is absent, i.e. they observed a change in the intensity emitted at the back facet of the LD [10]. They further demonstrated a primitive optical-data reader based on a rotating disc with cutouts placed in the external cavity [11]. Ukita et al. fabricated a laser device based on an extremely short strong-feedback external-cavity laser that can be used for optical-disc read out. The system uses self-mixing properties, and measures the change in light properties using an integrated PD [12], quite distinct from our approach which entirely dispenses with the PD. In [10] it was shown that the change in feedback can be sensed via the LD terminal voltage $V$ under constant-current $J$ operation. While it was suggested in [10] to monitor $V$ as the optical-data readout parameter, the experiment does not appear to have been carried out.

In this paper, we show $V$ can be used to measure the change in reflectivity of an object because when light is re-injected into an $\mathrm{LD}$, a drop in the previously stationary DC voltage occurs, as demonstrated in $[7,8]$. Thus, we can scan an object with an LD and measure the change in the DC terminal voltage $V$. Previously, twodimensional optical scanning and imaging based on the measurement of the voltage change induced by optical feedback was shown in [13]. The physical origin of this effect is the enhancement in stimulated emission, which results in a reduction of inversion because the voltage is proportional to the changes in the quasi-Fermi-level difference [8]. Essentially, the DC level observed is proportional to the bias current from the current controller. Therefore, the DC level is defined as the measured voltage averaged over a timescale that is short compared with the duration of an optical bit, but long compared with any intrinsic dynamics of the LD with constant optical feedback. The DC level only varies [without a spinning compact disc (CD)] with the fluctuations associated with the current controller. These factors together are on the order of $\mu V s$. Thus, large changes in the level of the DC voltage $(\sim 60 \mathrm{mV})$ are due to the effect we are exploiting.

Specifically, we make the following claims. (i) We read bits from a CD-ROM under realistic dynamical conditions via changes in the LD terminal voltage due to changes in the amount of optical intensity on reflection from the CD-ROM. (ii) The signals produced are compatible with the practical application of this effect in the context we focus on. (iii) The feedback mechanism is temporally incoherent, thus making the approach robust against mechanical vibrations, deflections, or small misalignments. Since the effect is temporally incoherent, it is distinct from that exploited for conventional self-mixing interferometry.

An LD package from a commercial CD-ROM drive was used in order to show the ability to read optical media and the simplicity of the approach. CD-ROM drives use an LD that emits light $(\lambda=780 \mathrm{~nm})$ that strikes the medium and is reflected or not depending on where the light strikes the CD-ROM (see below). The beam is then, if reflected, sent back into the laser package where there is typically a beam splitter (BS) that routes the light to a PD external to the laser. In our experiment, the BS was removed in order to route the reflected light back onto the LD (Fig. 1). This allowed for the imaging of the optical disc using as the contrast mechanism the variations in reflectivity of the optical disc or the encoded bits. To emphasise, we monitor only the DC component of the voltage across the LD and not dynamic components of the voltage.

\section{Experiments}

To demonstrate the basic principle first, a high-reflectivity mirror (Newport 10Z40DM.8) and digital multimeter were used to send the light back onto the LD and measure $V$, respectively. Next, a blazed diffraction grating with a gold coating designed for the nearinfrared with a blaze angle, $\theta=28^{\circ}$, and a grating pitch, $\Lambda=1.6 \mu \mathrm{m}$ was scanned using the LD from the CD-ROM drive (Samsung SM-308). Specifically, the LD package was rastered across the grating using an electric nano-positioning actuator (Newport NanoPZ) while simultaneously measuring the value of $V$ with a digital multimeter. This experimental setup can be seen in Fig. 2. 


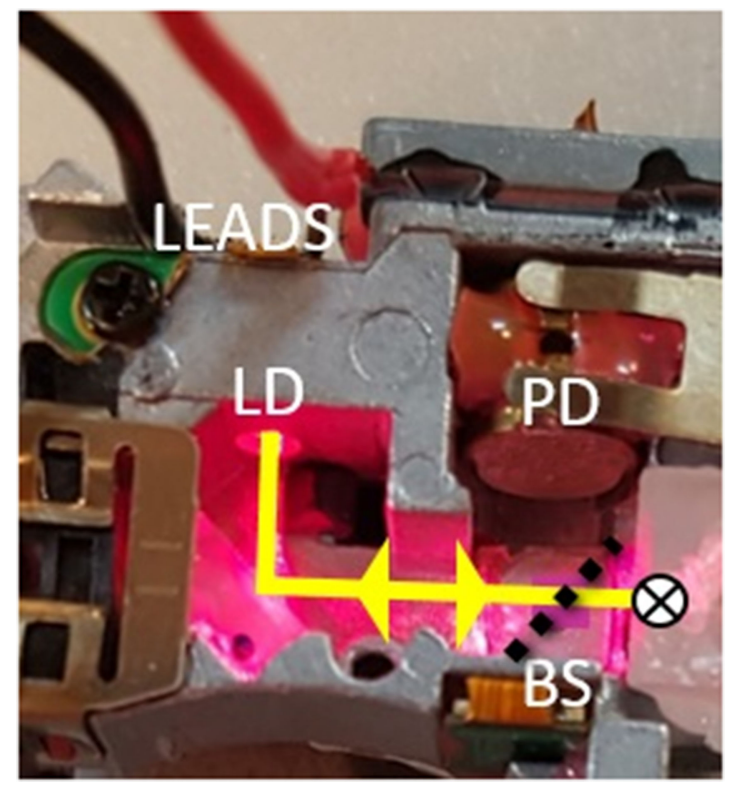

Fig. 1 Close-up of the LD package. BS and the dashed line represent the beam splitter. $L D$ and $P D$ stand for laser diode and photodiode, respectively. The shown beam path leaves the $L D$ and strikes a mirror at a $45^{\circ}$ angle and goes to another mirror which directs the beam through the page (marked by the symbol $x$ ) and onto the CD-ROM. The shown leads are soldered onto the $L D$ in order to provide power and a means for measuring $\checkmark$

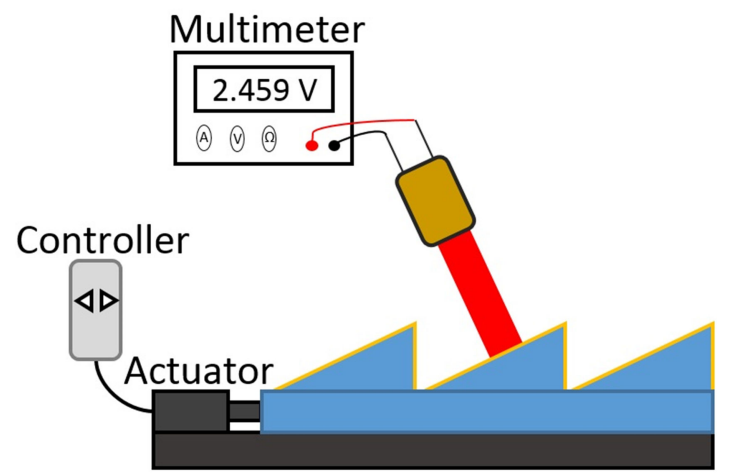

Fig. 2 Laser is incident on a gold-coated blazed diffraction grating with pitch $\Lambda=1.6 \mu \mathrm{m}$. A nano-positioning actuator is used to accurately raster the grating under the $L D$ while a digital multimeter measures $V$

Bits were then read from the CD-ROM by placing the modified LD package seen in Fig. 1 back into the CD-ROM drive, and placing a CD-ROM into the tray. A DC motor was used to spin the disc using a voltage source to control the rotational speed of the motor, as seen in Fig. 3. The rotational speed was measured by placing a line on the disc and using a fast video camera that films at $240 \mathrm{fps}$. By counting the number of frames it takes for the disc to rotate one time the linear relationship between voltage and rotations per minute (RPMs) was plotted. While the CD was spinning, $V$ was measured using an oscilloscope (Agilent DSO80804B) with a bandwidth of $12 \mathrm{GHz}$.

We estimated the coherence length in order to determine whether the feedback was temporally coherent or not. A measurement using an optical spectrum analyser showed a coherence length of around $8 \mathrm{~mm}$ as confirmed by Grattan and Meggitt [14]. Since the round-trip length of the external cavity is 3 $\mathrm{cm}$, the feedback is incoherent when it returns to the LD. It has been shown experimentally and numerically that incoherent feedback in systems has a direct effect on the carrier number and thus the terminal voltage $[15,16]$. As we discuss below, our approach therefore is distinct from self-mixing interferometry and thus is expected to exhibit superior mechanical stability.

\section{Results and discussion}

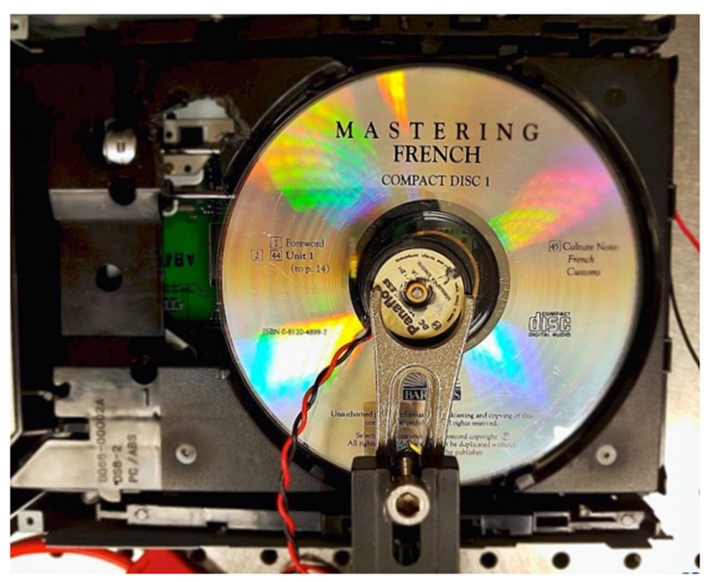

Fig. $3 C D-R O M$ in the modified $C D$ drive. The motor and holder are both visible, and the motor is controlled by a voltage source. The leads from the $L D$ can be seen on the right-hand side and go to an oscilloscope

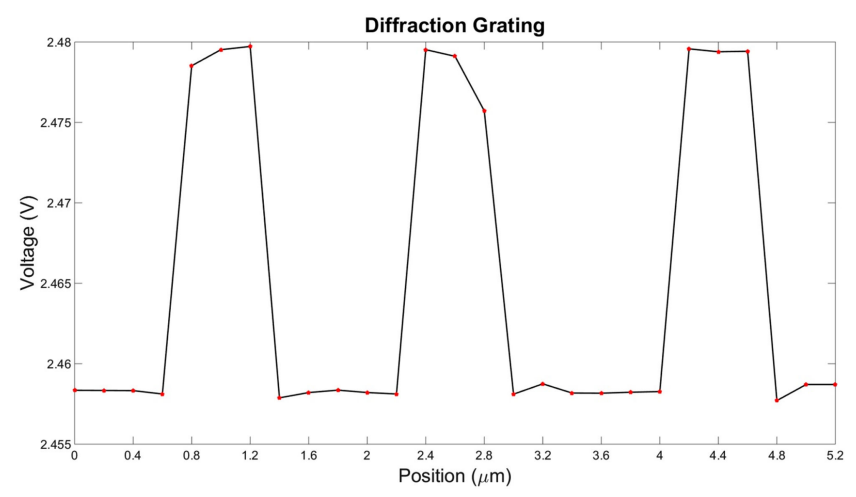

Fig. $4 V$ changes periodically with the same period as the grating, $1.6 \mu \mathrm{m}$

Table $1 \quad V$ in the presence or absence of two reflective objects, a mirror, and a diffraction grating. The value corresponding to feedback for the diffraction grating is when the laser spot is centred over a blazed surface giving maximum feedback

\begin{tabular}{lcc}
\hline & Feedback, V & No feedback, V \\
\hline mirror & 2.401 & 2.479 \\
diffraction grating & 2.459 & 2.479 \\
\hline
\end{tabular}

When the mirror was aligned, a reduction in $V$ of $0.078 \mathrm{~V}$ was observed (Table 1). The drop in $V$ suggests that amplification would not be needed and established that feedback could be achieved. The diffraction grating was scanned using the LD, which was positioned such that maximum reflectivity was obtained from the diffraction grating and with an incidence angle equal to the blaze angle. As seen in Table 1, the measured value of the voltage reduction for the diffraction grating was smaller than that of the mirror, but still sufficiently large to be measured without amplification. We changed the position of the diffraction grating in steps of $200 \mathrm{~nm}$ and the resulting value of $V$ as a function of position is shown in Fig. 4, as previously reported in [17].

The periodicity in Fig. 4 is due to the periodic nature of blazed gratings. When the light is incident perpendicular to a blaze, as seen in Fig. 5, feedback is achieved and a drop in the voltage is seen. When the light is at the drop off from one blaze to the next there is scattering and interference. The destructive interference occurs due to a phase shift for small values of $\theta$, where the phase difference is $\phi=4 \pi \Lambda \theta / \lambda$. For our experiment, the phase difference is $\phi \simeq 5 \pi$ which leads to nearly perfect destructive interference. We also note that we are able to see changes on an even smaller scale than $1.6 \mu \mathrm{m}$. Indeed, Fig. 4 shows that we are able to see variations in $V$ on a scale as small as $0.2 \mu \mathrm{m}$, which is significantly smaller than any distance one would need to detect on an optical disc. 


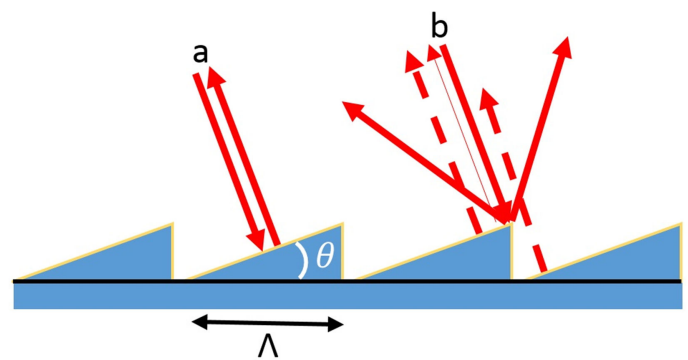

Fig. 5 Schematic cross-section of the blazed grating, which has a blaze angle, $\theta=28$, and grating pitch, $\Lambda=1.6 \mu \mathrm{m}$. For the rays incident at point $a$, there is a strong reflection leading to substantial feedback on the LD. Which is detected as a decrease in $V$. However, for light incident at point $b$ the reflection is weak due to scattering (solid) and destructive interference (dashed), leading to a smaller optical feedback and an increase in $V$

We next detected bits on a spinning CD-ROM. As can be seen in Fig. 6, bit changes are seen by monitoring $V$. Changes in the value of $V$ occur due to the modulation in reflectivity given by the CD-ROM as it spins. When light is incident on the change between a land and a pit or vice versa, there is near perfect destructive interference leading to negligible feedback on the laser and an increase in $V$, as illustrated in Fig. 7.

To further confirm that actual bits were seen, it was necessary to read and interpret them. CD-ROMs use an 8-14 bit channel conversion and have a sync header sequence consisting of 12 bytes (96 bits) every 2352 bytes [18]. Also, the 8-14 bit channel conversion is setup such that no other data sequence is possible that would have the same bit sequence as a header. It is known that every $1.51 \mathrm{~cm}$ of rotational distance on the disc a sync header should be found. The raw data from the oscilloscope was searched for headers, and it was experimentally determined that a header was occurring every $\sim 1.59 \pm 0.1 \mathrm{~cm}$ of rotational distance. The error in the estimation is due to the imperfect knowledge of the rotational speed of the disc and is within the estimating range. The measurement of the bits was repeated ten times for verification with consistent results. A statistical analysis of the chance of this header sequence occurring randomly one time reveals it is a $1 / 10^{28}$ chance. Experimentally, the correction header sequence was observed two times and was separated by approximately the same distance as theoretically calculated. Furthermore, the separations between ones are exactly proportional to the number of zeros within the error of the sampling rate. The previous observations confirm unambiguously our ability to detect the bits on a CDROM. Also of note, using the DC motor we were able to achieve speeds at the level of an actual CD-ROM drive.

We have determined the signal-to-noise ratio (SNR) for our system to be $\sim 9 \mathrm{~dB}$. In comparison, the SNR for the PD is known to be poor without additional pre-processing including a current amplifier, a filter, and a transimpedance amplifier. After the complex circuitry, the PD has a SNR of $\sim 47 \mathrm{~dB}$ [19]. Although the PD may have a better SNR after circuitry, in a binary system if the value of a one is significantly different than a zero, the system will work. A qualitative analysis of the waveforms in Fig. 6 shows that we are able to distinguish between binary 0's and 1's with a simple thresholding and without any added circuitry.

A second consideration is power. The difference in electrical power delivered by the terminal voltage probes is given by the product of the voltage difference between 0 and $1(\sim 0.08 \mathrm{~V}$ from our measurements suggesting that this could be implemented in applications without amplification) and the drive current $50 \mathrm{~mA}$. The resulting power is $\sim 4 \mathrm{~mW}$. This power level is larger than that produced by the $\mathrm{PD}$ in a conventional CD-ROM drive with a change in power of $\sim 10-100 \mu \mathrm{W}$.

We also comment on the temporal coherence of the feedback due to the relatively long external-cavity round-trip time and the relatively short coherence time of the LD (We emphasise that the spatial coherence across the laser beam is high and important as this provides the contrast mechanism.). The temporal incoherence implies that the population principle is insensitive to variations in the external-cavity length on the order of the optical wavelength. The same is not the case for a coherent system, which is susceptible to vibration. Indeed, in our demonstration, no additional mechanical stabilisation or alignment, beyond the stock CD-ROM drive housing, was provided.

In traditional CD-ROM technology, an array of PDs is used. There are different topologies between varying manufacturers, but in all of the configurations that we are aware, one or more PDs are utilised for measuring the light being fed back by the CD for data, and other PDs are used for tracking and focusing [19]. Thus, a new array would need to be fabricated with one LD and PDs on each side of the laser. These PDs would be utilised for tracking and focusing just as is currently done in $\mathrm{CD}$ systems. However, nonetheless the sensing portion would still be greatly simplified and improved by utilising the approach in this paper.

\section{Conclusion}

We have shown that we are able to image variations in the surface of reflective objects by monitoring the DC value of the laser voltage $V$. This has allowed us for the first time to our knowledge to read the bits on a CD-ROM simply using an LD and without a PD. The significant variation in between ones and zeros seen in our waveforms imply this can be integrated in a system without amplification. Our approach also simplifies system architecture by eliminating the BS, PD, and the circuitry associated with the PD. In addition to optical-disc reading, applications where the PD is difficult to accommodate due to geometrical constraints or in other

Start of Header Sequence
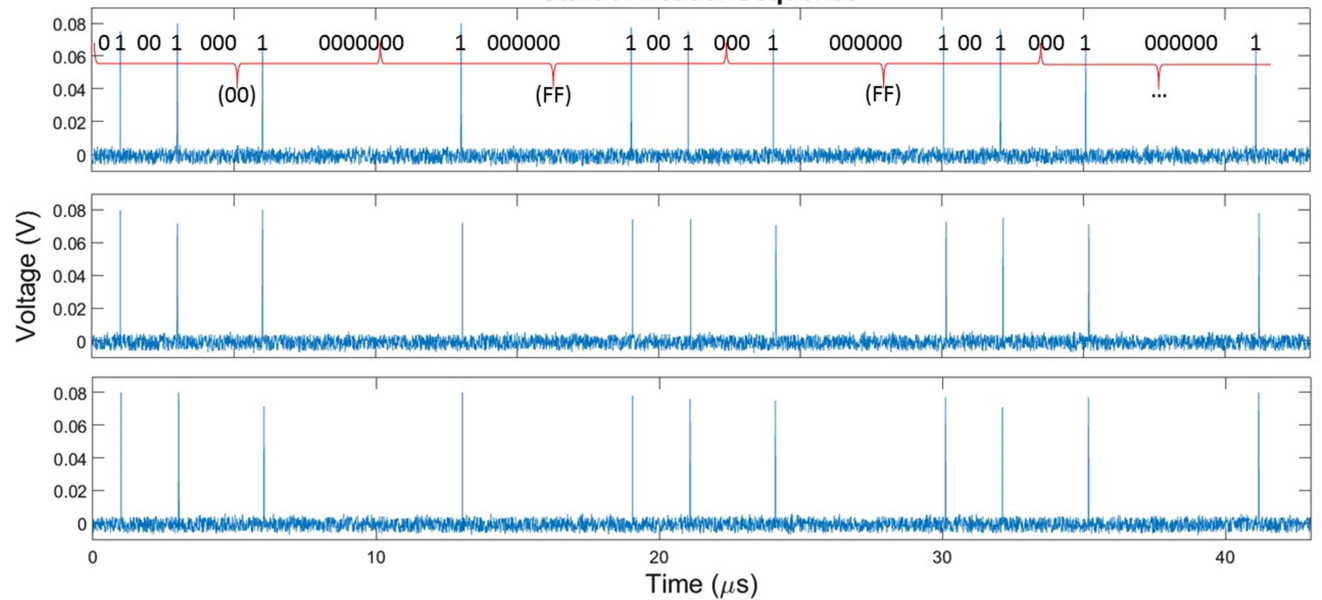

Fig. $6 \mathrm{~V}$ as a function of time with a spinning CD-ROM (three different readings). The beginning portion of a header is shown. The total header consists of 12 bytes (00 FF FF FF FF FF FF FF FF FF FF 00). After the 8-14 bit conversion, 00 becomes (01001000100000), and FF becomes (00100000010010) [18]. A one is encoded as a change in height on the disc 


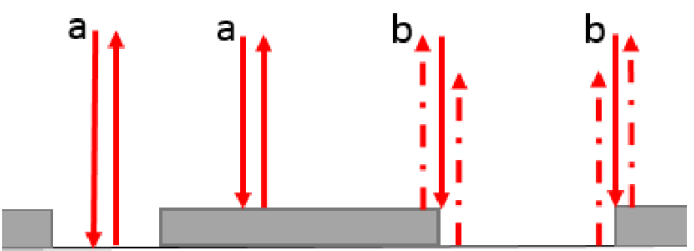

Fig. 7 Schematic cross-section of a CD-ROM disc. When the light is incident at a surface other than an edge the light is reflected back into the $L D$ leading to feedback and a drop in $V$ (rays labelled a). When light is incident on a transition from a land to a pit the light interferes destructively, because the distance between the land and pit is $\lambda / 4$ corresponding to a phase shift equal to $\pi$, as seen by the rays labelled $b$

portions of the spectrum where the detector is expensive such as in the terahertz range may benefit from the approach developed in this paper. The approach in general relies on a feedback time that exceeds the LD coherence time and a sufficiently high-reflectivity contrast between at least two states.

\section{Acknowledgments}

We gratefully acknowledge helpful discussion with Cedric Pradalier and the financial support of the Conseil Régional du Grand Est and the Fonds Européen de Développement Régional (FEDER).

\section{References}

[1] Rudd, M.J.: 'A laser Doppler velocimeter employing the laser as a mixeroscillator', J. Phys. E, 1996, 1, (1), p. 723

[2] Shinohara, S., Mochizuki, A., Yoshida, H., et al.: 'Laser Doppler velocimeter using the self-mixing effect of a semiconductor laser diode', Appl. Opt., 1986, 25, (25), pp. 1417-1419

[3] Donati, S., Giuliani, G., Merlo, S.: 'Laser diode feedback interferometer for measurement of displacements without ambiguity', IEEE J. Quantum Electron., 1995, 31, (31), pp. 113-119
[4] Quotb, A., Ramirez-Miquet, E.E., Tronche, C., et al.: 'Optical feedback interferometry sensor for flow characterization inside ex-vivo vessel'. IEEE Sensors, Valencia, November 2014, pp. 362-365

[5] Lacot, E., Day, R., Pinel, J., et al.: 'Laser relaxation-oscillation frequency imaging', Opt. Lett., 2001, 26, (19), p. 1483

[6] Morikawa, T., Mitsuhashi, Y., Shimada, J., et al.: 'Return-beam-induced oscillations in self-coupled semiconductor lasers', Electron. Lett., 1976, 12, (12), pp. 435-436

[7] Kazarinov, R.F., Suris, R.: 'Heterodyne reception of light by an injection laser', Sov. Phys.-JETP, 1974, 66, (39), pp. 522-527

[8] Sahai, A.A., Kim, B., Choi, D., et al.: 'Mapping the nonlinear dynamics of a laser diode via its terminal voltage', Opt. Lett., 2014, 39, (39), pp. 5630-5633

[9] Chang, C.Y, Choi, D., Locquet, A et al. 'A multi-GHz chaotic optoelectronic oscillator based on laser terminal voltage', Appl. Phys. Lett. 2016, 19, (108), p. 191109

[10] Mitsuhashi, Y., Morikawa, T., Sakura, K., et al:: 'Self-coupled optical pickup', Opt. Commun., 1976, 17, (17), pp. 95-97

[11] Seko, A., Mitsuhashi, Y., Morikawa, T., et al.: 'Self-quenching in semiconductor lasers and its applications in optical memory readout', Appl. Phys. Lett., 1975, 27, (27), pp. 140-141

[12] Ukita, H., Uenishi, Y., Katagiri, Y.: 'Applications of an extremely short strong-feedback configuration of an external-cavity laser diode system fabricated with GaAs-based integration technology', Appl. Opt., 1994, 33 , (33), pp. 5557-5563

[13] Heinis, D., Gorecki, C., Bargiel, S., et al.: 'Feedback-induced voltage change of a vertical-cavity surface-emitting laser as an active detection system for miniature optical scanning probe microscopes', Opt. Express, 2006, 14, (14), pp. 3396-3405

[14] Grattan, K., Meggitt, B.T.: 'Optical fiber sensor technology' (Chapman and Hall, London, 1995)

[15] Solorio, J.S., Sukow, D.W., Hicks, D.R., et al: 'Bifurcations in a semiconductor laser subject to delayed incoherent feedback', Opt. Commun., 2002, 214, (214), pp. 327-334

[16] Rogister, F., Locquet, A., Pieroux, D., et al:: 'Secure communication scheme using chaotic laser diodes subject to incoherent optical feedback and incoherent optical injection', Opt. Lett., 2001, 26, (26), pp. 1486-1488

[17] Wishon, M.J., Mourozeau, G., Ng, K., et al.: 'Reading a CD-ROM without a photodiode'. Proc. SPIE 9892 Conf. Semiconductor Lasers and Laser Dynamics VII, May 2016

[18] 'Data interchange on read-only $120 \mathrm{~mm}$ optical data disks (CD-ROM)' (European Computer Manufacturers Association, 1996)

[19] Stan, S.G.: 'The CD-ROM drive: a brief system description' (Spring-Science + Business Media, Boston, MA, 1998) 